\title{
Сферический распределенный брэгговский отражатель со всенаправленной стоп-зоной в ближней ИК-области спектра
}

\author{
(C) А.В. Медведев, А.А. Дукин, Н.А. Феоктистов, В.Г. Голубев \\ Физико-технический институт им. А.Ф. Иоффре Российской академии наук, \\ 194021 Санкт-Петербург, Россия \\ E-mail: medvedev@gvg.ioffe.ru
}

Поступила в Редакцию 7 фревраля 2019 г.

В окончательной редакции 10 февраля 2019 г.

Принята к публикации 14 фревраля 2019 г.

\begin{abstract}
Методом плазмохимического газофазного осаждения созданы сферические распределенные брэгговские отражатели (СРБО) на ближний инфракрасный диапазон спектра. СРБО состоят из чередующихся четвертьволновых слоев $a-\mathrm{Si}: \mathrm{H}$ и $a-\mathrm{SiO}_{2}$, нанесенных на стеклянную или кварцевую микросферу диаметром 500 мкм, легированную ионами эрбия. Измерены спектры отражения и пропускания от СРБО в разных точках его поверхности. Зарегистрирована широкая полоса с высоким коэффициентом отражения и малым коэффициентом пропускания - стоп-зона. Продемонстрировано, что для разных радиальных направлений из центра микросферы стоп-зоны перекрываются, образуя всенаправленную стоп-зону. Исследовано влияние всенаправленной стоп-зоны на спонтанную эмиссию ионов эрбия (1.53 мкм) из кварцевой микросферы. Показано, что всенаправленная стоп-зона подавляет интенсивность спонтанной эмиссии более чем на порядок величины.
\end{abstract}

Ключевые слова: сферический распределенный брэгговский отражатель, всенаправленная стоп-зона, ближний инфракрасный диапазон, аморфный кремний, эрбий, подавление спонтанной эмиссии, длина волны телекоммуникации 1.5 мкм.

DOI: 10.21883/FTP.2019.07.47867.9077

\section{1. Введение}

Сферический распределенный брэгговский отражатель (СРБО) состоит из периодически чередующихся четвертьволновых слоев с высоким и низким показателем преломления, которые нанесены на прозрачную диэлектрическую сферу (ядро) [1,2]. В спектрах отражения и пропускания СРБО имеются стоп-зоны, т. е. спектральные области, запрещенные для распространения электромагнитных волн. Вследствие сферической симметрии стоп-зоны для радиальных направлений распространения света спектрально перекрываются и образуют всенаправленную стоп-зону. Важным преимуществом СРБО является то, что в них всенаправленная стопзона возникает при любом значении оптического контраста - отношения показателей преломления слоев.

СРБО со всенаправленной стоп-зоной могут найти применение для управления спонтанной эмиссией [3-5], для создания низкопороговых лазеров [6] и однофотонных источников света [6-8]. Однако, несмотря на большой потенциал практического применения, существует мало работ, посвященных созданию и экспериментальному исследованию их оптических свойств. Это обусловлено трудностью воспроизводимого нанесения на микроскопическую сферическую частицу тонких (толщиной порядка нескольких десятков-сотен нанометров) однородных по химическому составу и толщине слоев. К настоящему времени предложено несколько способов создания СРБО: электронно-лучевое осаждение $\mathrm{ZnS} / \mathrm{Na}_{3} \mathrm{AlF}_{6}$ [9], травление в сочетании с химическим газофазным осаждением
$\mathrm{Si} / \mathrm{SiO}_{2}$ [3], многостадийная эмульсионная полимеризация полистирола/поли(трифторэтил метакрилата) [10]. В нашей предыдущей работе [11] была продемонстрирована применимость для изготовления СРБО метода плазмохимического газофазного осаждения (plasmaenhanced chemical vapor deposition - PECVD).

Цель настоящей работы заключается в изготовлении СРБО, обладающего широкой всенаправленной стопзоной в ближнем ИК-диапазоне, методом PECVD и в исследовании его оптических свойств, в том числе влияния образующейся всенаправленной стоп-зоны на люминесценцию ионов эрбия, введенных в микросферу, на которую нанесен СРБО. В качестве материала слоев СРБО выбраны гидрогенизированный аморфный кремний $(a-\mathrm{Si}: \mathrm{H})$ и аморфный оксид кремния $\left(a-\mathrm{SiO}_{2}\right)$. Эти материалы прозрачны в ближнем ИК-диапазоне спектра и обладают большим оптическим контрастом, что обеспечивает получение широкой стоп-зоны.

\section{2. Эксперимент}

С целью исследований оптических свойств СРБО, в качестве прозрачной диэлектрической сферы для СРБО использовались микросферы из силикатного натриевокальциевого стекла диаметром 500 мкм. Для исследований влияния всенаправленной стоп-зоны СРБО на спонтанную эмиссию использовались кварцевые микросферы диаметром 500 мкм, легированные ионами эрбия $\mathrm{Er}^{3+}$. Эти микросферы выплавлялись из оптического одномодового волокна ER110-4/125 фирмы Thorlabs с исходной концентрацией эрбия, равной $6 \cdot 10^{19} \mathrm{~cm}^{-3}$. 


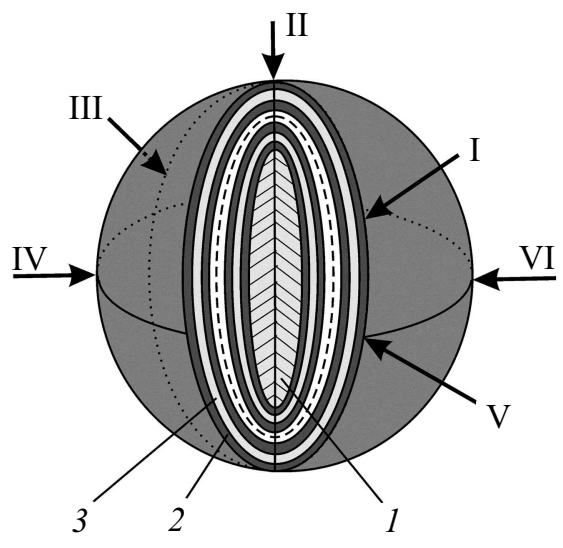

Рис. 1. Схематическое изображение СРБО, нанесенного на прозрачную диэлектрическую микросферу: 1 - прозрачная микросфера из стекла или кварца, легированного $\mathrm{Er}^{3+}, 2-$ четвертьволновый слой $a$ - $\mathrm{Si}: \mathrm{H}, 3$ - четвертьволновый слой $a-\mathrm{SiO}_{2}$. Пунктиром обозначена часть слоев, которая опущена на рисунке. I, II, III, IV, V, VI - точки, в которых зарегистрированы спектры отражения, приведенные на рис. 2. Жирными стрелками показаны направления лучей. Точки I, II, III лежат на меридиане СРБО через $45^{\circ}$, точки IV, V, VI лежат на экваторе СРБО через $90^{\circ}$.

СРБО состоит из 11 чередующихся четвертьволновых слоев $a-\mathrm{Si}: \mathrm{H}$ и $a-\mathrm{SiO}_{2}$, нанесенных на микросферу (рис. 1). Слои $a-\mathrm{Si}: \mathrm{H}$ и $a-\mathrm{SiO}_{2}$ наносились методом PECVD в едином технологическом цикле без экспозиции на воздухе в промежутках между последовательными стадиями роста отдельных слоев структуры. Чередование слоев обеспечивалось переключением газовых потоков. Слои $a-\mathrm{Si}: \mathrm{H}$ осаждались из $10 \%$ смеси силана с аргоном. При росте слоев $a-\mathrm{SiO}_{2}$ соотношение потоков силана и кислорода составляло $\sim 1: 1$. Другие параметры процесса PECVD были следующие: рабочее давление в реакторе составило 0.1-0.2 Торр, частота высокочастотного излучения - 17 МГц, высокочастотная мощность $-0.03-0.1 \mathrm{Bт} / \mathrm{cm}^{2}$, температура подложки - 200-250 ${ }^{\circ}$, суммарный поток газовой смеси $5-10$ норм. $\mathrm{cm}^{3} /$ мин ( $\left.\mathrm{sccm}\right)$. Использованная технология позволяет наносить тонкие однородные слои $a-\mathrm{Si}: \mathrm{H}$ и $a-\mathrm{SiO}_{2}$ на микросферы с диаметром от единиц до десятков и сотен микрон. Толщина слоев $a-\mathrm{Si}: \mathrm{H}(\sim 110 \mathrm{Hм})$ и $a-\mathrm{SiO}_{2} \quad(\sim 220 \mathrm{Hм})$ выбиралась такой, чтобы центр первой стоп-зоны $\left(\lambda_{0}\right)$ находился в ближней ИК-области спектра ( 1400 нм), и первая стоп-зона включала в себя длину волны 1530 нм.

Фотолюминесценция (ФЛ) ионов эрбия $\mathrm{Er}^{3+}$ возбуждалась диодным инфракрасным лазером с длиной волны 980 нм мощностью 400 мВт. Спектры отражения, пропускания и ФЛ измерялись по методике, описанной в [11], с использованием микрообъектива с фокусным расстоянием 5 мм и объектива с фокусным расстоянием 50 мм. Спектры регистрировались по нормали к поверхности СРБО, вдоль экватора микросферы через 90 и вдоль меридиана через $45^{\circ}$.

\section{3. Результаты и обсуждение}

Характерные спектры отражения от разных точек поверхности СРБО со стеклянным ядром приведены на рис. 2. Положение точек, в которых записывались спектры, и направления лучей обозначены на рис. 1 стрелками I, II, III, IV, V, VI. Во всех исследованных спектрах в области длин волн $\sim 1200-1700$ нм наблюдалась широкая полоса отражения (первая стоп-зона), с центром $\lambda_{0}$ в диапазоне 1300-1400 нм. Эта полоса отражения, как и в планарных распределенных брэгговских отражателях (РБО) [12,13] и микрорезонаторах [14-16], возникает за счет брэгговского отражения от группы слоев с периодически меняющимся показателем преломления. В этой области коэффициент отражения от СРБО близок к единице. За коротковолновым краем стоп-зоны в спектрах отражения наблюдается сложная интерференционная картина. Граница измерения спектров 1700 нм определяется границей чувствительности использованного фотодетектора. Спектральное положение стоп-зоны незначительно смещается при регистрации отражения в

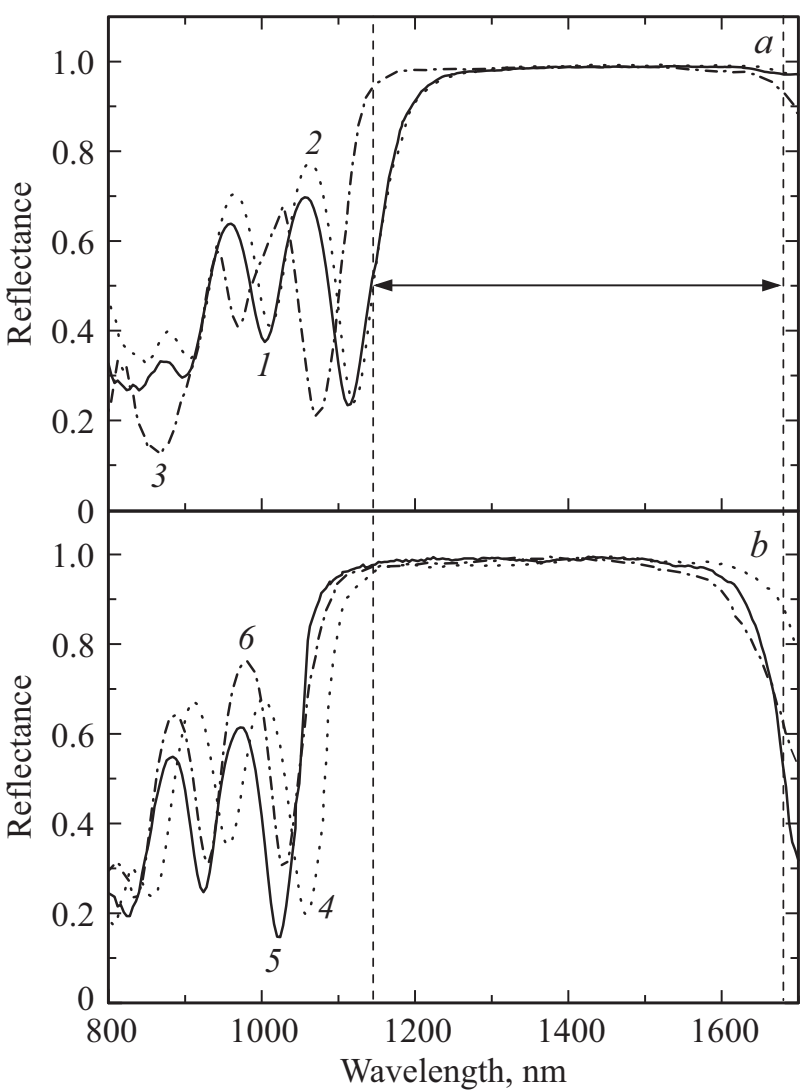

Рис. 2. Спектры отражения света от СРБО. $a$ - спектры 1,2, 3 зарегистрированы в точках I, II, III, лежащих на меридиане СРБО (рис. 1); $b$ - спектры 4, 5, 6 зарегистрированы в точках IV, V, VI, лежащих на экваторе СРБО (рис. 1). Спектры нормированы на максимум коэффициента отражения. Горизонтальная двусторонняя стрелка показывает область перекрытия полос брэгговского отражения на уровне половины высоты максимума отражения. Вертикальные штриховые линии показывают границы области перекрытия. 


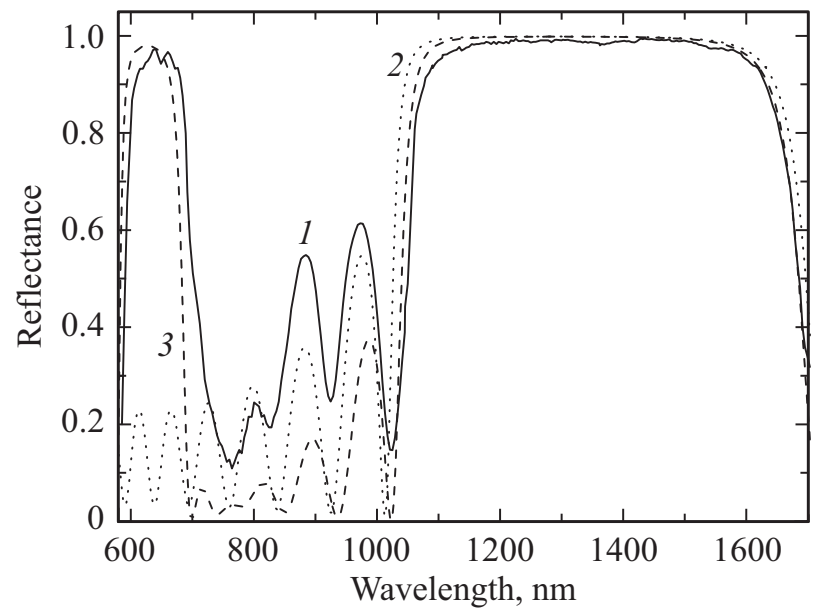

Рис. 3. Спектры отражения света от СРБО. 1 - экспериментальный спектр отражения, зарегистрированный в точке $\mathrm{V}$ на рис. 1; 2 - спектр отражения, рассчитанный при условии, что все оптические толщины слоев равны четверти длины волны $\lambda_{0} ; 3-$ рассчитанный спектр отражения от СРБО, в котором параметры слоев подобраны таким образом, чтобы рассчитанная форма полосы брэгговского отражения в области первой стоп-зоны $(1040-1680$ нм $)$ максимально совпала с наблюдаемой экспериментально в спектре 1. Спектры нормированы на максимум коэффициента отражения.

разных точках поверхности СРБО. Большой оптический контраст слоев $a-\mathrm{Si}: \mathrm{H}$ и $a-\mathrm{SiO}_{2}$ обусловливает большую ширину зарегистрированных стоп-зон и тем самым обеспечивает широкий диапазон их спектрального перекрытия при измерениях в разных точках на поверхности. Область перекрытия стоп-зон на уровне половины высоты максимума отражения (1140-1680 нм) показана горизонтальной двусторонней стрелкой на рис. 2,a. Перекрытие стоп-зон для различных радиальных направлений из центра микросферы свидетельствует о том, что в исследованном образце существует всенаправленная стоп-зона. Она включает в себя длины волн 1260-1625 нм, используемые в волоконно-оптических линиях связи [17]. Экспериментально зарегистрированная всенаправленная стоп-зона имеет центр на длине волны 1410 нм и относительную ширину $\sim 0.38$ (отношение ширины к центру стоп-зоны).

Экспериментальный спектр отражения света от СРБО (кривая 1 на рис. 3) сопоставлен со спектром, рассчитанным методом матриц переноса [15] при условии, что все оптические толщины слоев точно равны четверти длины волны $\lambda_{0}$ (кривая 2 на рис. 3). Расчет проведен в приближении, когда участок поверхности СРБО с малой площадью описывается системой плоскопараллельных слоев. Такое приближение оправданно, так как сигнал отражения регистрировался с участка поверхности диаметром $\sim 5$ мкм, что намного меныше диаметра сферы 500 мкм, и в этом случае сферичностью поверхности можно пренебречь. Видно, что рассчитанная ширина первой стоп-зоны больше, чем регистрируемая экспериментально. Кроме того, в экспериментальном спектре присутствует вторая стоп-зона (полоса с высоким коэффициентом отражения в диапазоне 590-700 нм), которая отсутствует в рассчитанном спектре.

Это можно объяснить, если принять во внимание, что вследствие возможных несовершенств технологического процесса оптические толщины слоев отклоняются от заданных четвертьволновых значений. Варьируя параметры слоев, удалось подогнать рассчитанную форму спектра (кривая 3 на рис. 3) в области первой стопзоны к экспериментально наблюдаемой. Дополнительным свидетельством правильности проведенной подгонки является наличие в расчетном спектре второй стопзоны приблизительно на тех же длинах волн, что и в экспериментальном. Проведенная подгонка позволила уточнить толщины слоев и значение показателя преломления $a-\mathrm{Si}: \mathrm{H}(n \approx 2.83)$.

Для идеального СРБО, в котором все оптические толщины слоев точно равны четверти длины волны $\lambda_{0}$, спектральное положение и ширина всенаправленной стоп-зоны совпадают со спектральным положением и шириной стоп-зоны для плоского РБО с теми же параметрами слоев. Поэтому относительную ширину всенаправленной стоп-зоны можно рассчитать, используя формулу для относительной ширины $\left(\Delta \lambda / \lambda_{0}\right)$ первой стоп-зоны плоского РБО [18]:

$$
\Delta \lambda / \lambda_{0}=(4 / \pi) \arcsin \left(\left|n_{1}-n_{2}\right| /\left(n_{1}+n_{2}\right)\right),
$$

где $n_{1}$ и $n_{2}$ - показатели преломления слоев РБО. Используя значение показателя преломления $n(a-\mathrm{Si}: \mathrm{H}) \approx 2.83$ и $n\left(a-\mathrm{SiO}_{2}\right) \approx 1.45$, мы оценили максимально возможную относительную ширину всенаправленной стоп-зоны для СРБО, состоящего из четвертьволновых слоев $a-\mathrm{Si}: \mathrm{H}$ и $a-\mathrm{SiO}_{2}: \Delta \lambda / \lambda_{0}=0.42$. Полученное в эксперименте значение относительной ширины всенаправленной стоп-зоны (0.38) всего на 8.6\% меньше максимально возможного, что свидетельствует о хорошем качестве СРБО. Меньшее значение относительной ширины всенаправленной стоп-зоны в эксперименте можно объяснить отклонением толщин слоев от четвертьволновых значений и вариацией толщины слоев вдоль поверхности слоев СРБО.

В работе также исследовано влияние всенаправленной стоп-зоны СРБО на ФЛ ионов эрбия $\mathrm{Er}^{3+}$ в микросфере. На рис. 4 показаны спектры пропускания и ФЛ для СРБО с кварцевым ядром, легированным $\mathrm{Er}^{3+}$, записанные в трех разных точках поверхности. В области длин волн больше 1200 нм в спектрах пропускания (кривые 1-3) имеется широкая полоса малого пропускания - первая стоп-зона. Регистрируемая область спектрального перекрытия стоп-зон - всенаправленная стоп-зона - находится в диапазоне 1200-1700 нм. Длинноволновый край стоп-зон расположен за границей чувствительности фотоприемника.

Спектры ФЛ зарегистрированы в тех же точках на поверхности СРБО, что и спектры пропускания (кривые 4-6 на рис. 4). В спектрах ФЛ имеется полоса излучения $\mathrm{Er}^{3+}$ с максимумом на длине волны 


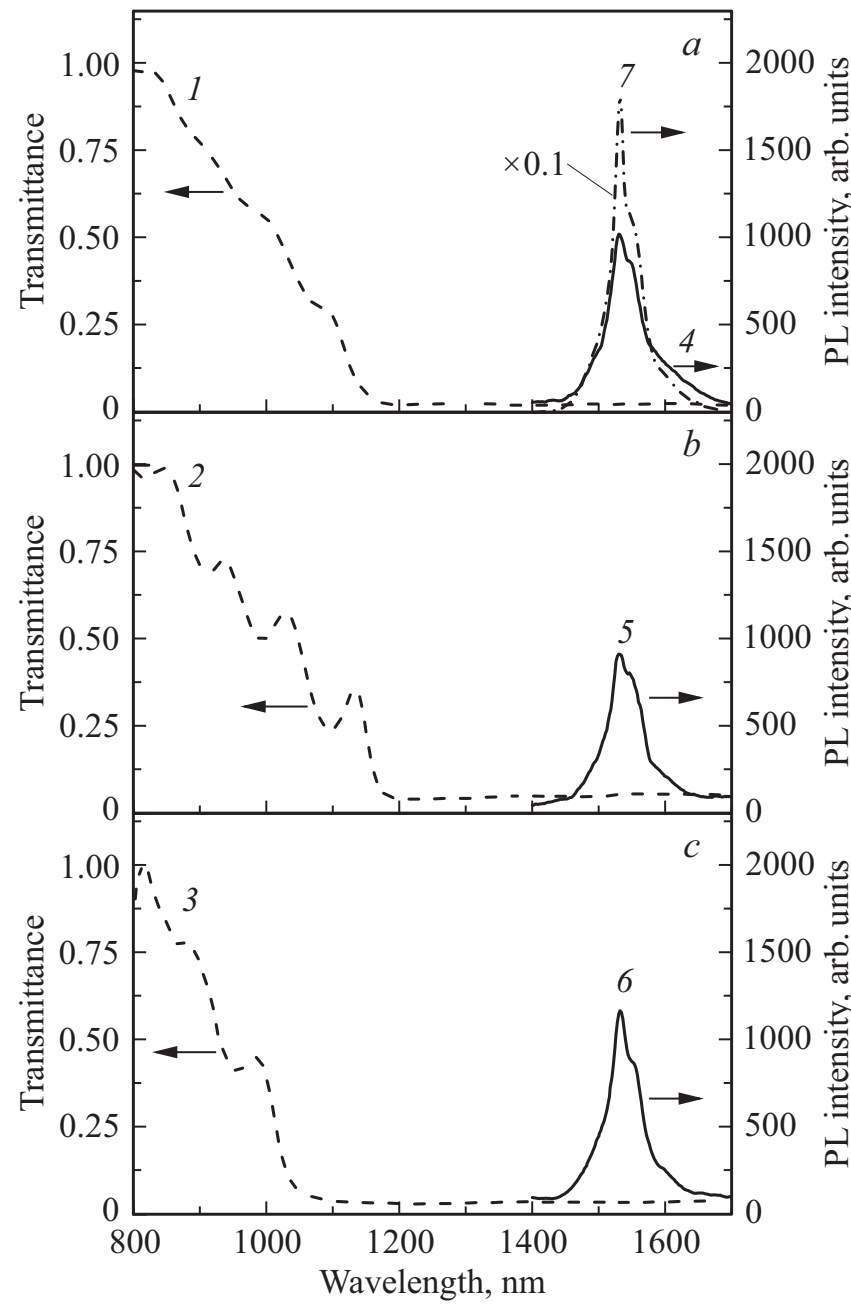

Рис. 4. 1,2,3 - спектры пропускания СРБО с кварцевой микросферой, легированной эрбием, зарегистрированные в точках II, III, IV этой микросферы (рис. 1); 4, 5, 6 - спектры ФЛ ионов эрбия из кварцевой микросферы, покрытой СРБО, зарегистрированные в тех же точках, что и спектры пропускания; 7 - спектр ФЛ ионов эрбия из кварцевой микросферы без СРБО, уменьшенный в 10 раз.

$\sim 1.53$ мкм, соответствующая внутрицентровому переходу ${ }^{4} I_{13 / 2} \rightarrow{ }^{4} I_{15 / 2}[14,15]$, которая полностью находится внутри всенаправленной стоп-зоны (рис. 4). Во всех спектрах интенсивность ФЛ ионов эрбия из кварцевой микросферы, покрытой СРБО в 15-20 раз меньше, чем интенсивность ФЛ ионов эрбия из кварцевой микросферы без СРБО (кривая 7 на рис. 4,a). Таким образом, показано, что всенаправленная стоп-зона подавляет спонтанную эмиссию ионов $\mathrm{Er}^{3+}$ на длине волны 1.53 мкм более чем на порядок величины.

\section{4. Заключение}

Методом плазмохимического газофазного осаждения изготовлены СРБО на ближний инфракрасный диапазон спектра (1140-1680 нм). СРБО состоят из 11 череду- ющихся четвертьволновых слоев гидрогенизированного аморфного кремния $(a-\mathrm{Si}: \mathrm{H})$ и аморфного оксида кремния $\left(a-\mathrm{SiO}_{2}\right)$, нанесенных на стеклянную или кварцевую микросферу диаметром 500 мкм. Кварцевые микросферы легированы эрбием, трехвалентные ионы которого излучают на длине волны 1.53 мкм. Созданные СРБО обладают высоким оптическим контрастом: отношение показателей преломления $n(a-\mathrm{Si}: \mathrm{H}) / n\left(a-\mathrm{SiO}_{2}\right) \approx 2$. Измерены спектры отражения и пропускания света от СРБО в разных точках его поверхности. Продемонстрировано, что СРБО обладает всенаправленной стоп-зоной в области 1140-1680 нм. Этот диапазон включает в себя длины волн, используемые в волоконно-оптических линиях связи. Спектры отражения от СРБО сопоставлены со спектрами, рассчитанными методом матриц переноса, в приближении, когда выбранный участок СРБО с малой площадью поверхности описывается системой плоскопараллельных слоев. Экспериментально исследованы спектры ФЛ ионов $\mathrm{Er}^{3+}$ из кварцевой микросферы, на которую нанесен СРБО. Показано, что интенсивность линии излучения ионов $\mathrm{Er}^{3+}$ (1.53 мкм), при попадании в область всенаправленной стоп-зоны, подавляется в 15-20 раз по сравнению с интенсивностью ФЛ эрбия из кварцевой микросферы без СРБО.

\section{Финансирование работы}

Работа выполнена в рамках государственного задания по теме № 0040-2019-0012.

\section{Конфликт интересов}

Авторы заявляют, что у них нет конфликта интересов.

\section{Список литературы}

[1] E.-X. Ping. J. Appl. Phys., 76 (11), 7188 (1994).

[2] K.G. Sullivan, D.G. Hall. Phys. Rev. A, 50 (3), 2701 (1994).

[3] Y. Xu, W. Liang, A. Yariv, J.G. Fleming, S. Lin. Opt. Lett., 28 (22), 2144 (2003).

[4] K.G. Sullivan, D.G. Hall. Phys. Rev. A, 50 (3), 2708 (1994).

[5] K. Imakita, H. Shibata, M. Fujii, S. Hayashi. Opt. Express, 21 (9), 10651 (2013).

[6] Y. Xu, W. Liang, A. Yariv, J.G. Fleming, S. Lin. Optics. Lett., 29 (5), 424 (2004).

[7] T.A. Nguyen, H.T. Dung. Phys. Rev. A, 76 (3), 033831 (2007).

[8] R. Shugayev, P. Bermel. Appl. Phys. Lett., 109 (22), 221102 (2016).

[9] C. Deumié, Ph. Voarino, C. Amra. Appl. Optics, 41 (16), 3299 (2002).

[10] I. Gourevich, L.M. Field, Z. Wei, C. Paquet, A. Petukhova, A. Alteheld, E. Kumacheva, J.J. Saarinen, J.E. Sipe. Macromolecules, 39 (4), 1449 (2006).

[11] А.В. Медведев, А.А. Дукин, Н.А. Феоктистов, В.Г. Голубев. Письма ЖТФ, 43 (19), 35 (2017).

[12] В.Г. Голубев, А.А. Дукин, А.В. Медведев, А.Б. Певцов, А.В. Селькин, Н.А. Феоктистов. ФТТ, 46 (10), 1756 (2004).

[13] M.V. Rybin, A.V. Zherzdev, N.A. Feoktistov, A.B. Pevtsov. Phys. Rev. B, 95 (16), 165118 (2017). 
[14] A.A. Dukin, N.A. Feoktistov, V.G. Golubev, A.V. Medvedev, A.B. Pevtsov, A.V. Sel'kin. Appl. Phys. Lett., 77 (19), 3009 (2000).

[15] В.Г. Голубев, А.А. Дукин, А.В. Медведев, А.Б. Певцов, А.В. Селькин, Н.А. Феоктистов. ФТП, 35 (10), 1266 (2001).

[16] A.A. Dukin, N.A. Feoktistov, V.G. Golubev, A.V. Medvedev, A.B. Pevtsov, A.V. Sel'kin. Phys. Rev. E, 67 (4), 046602 (2003).

[17] P.J. Winzer, D.T. Neilson, A.R. Chraplyvy. Opt. Express, 26 (18), 24190 (2018).

[18] G. Panzarini, L.C. Andreani, A. Armitage, D. Baxter, M.S. Skolnick, V.N. Astratov, J.S. Roberts, A.V. Kavokin, M.R. Vladimirova, M.A. Kaliteevski. ФTT, 41 (8), 1337 (1999).

Редактор Г.А. Оганесян

\title{
A spherical distributed Bragg reflector with omnidirectional stop-band in the near infrared region
}

\author{
A.V. Medvedev, A.A. Dukin, N.A. Feoktistov, \\ V.G. Golubev \\ loffe Institute, \\ 194021 St. Petersburg, Russia
}

Abstract Spherical distributed Bragg reflectors (SDBR) for near-infrared applications are elaborated via plasma-enhanced chemical-vapor deposition. They consist of the alternating quarter-wave layers of amorphous hydrogenized silicon $(a-\mathrm{Si}: \mathrm{H})$ and amorphous silicon oxide $\left(a-\mathrm{SiO}_{2}\right)$ deposited onto a glass microsphere or quartz microsphere, doped by erbium ions with a diameter of $500 \mu \mathrm{m}$. The reflection and transmission spectra of the SDBR are measured at different points of its surface. A wide band with a high reflectance and a low transmittance - a stop-band is detected. It is demonstrated that for different radial directions from the center of the microspheres the stop-bands overlap, forming an omnidirectional stop-band. The influence of the omnidirectional stop-band on spontaneous emission of erbium ions $(1.53 \mu \mathrm{m})$ from the quartz microsphere is studied. It is shown that the omnidirectional stop-band suppresses the intensity of spontaneous emission by more than an order of magnitude. 